\title{
Finite Temperature and Density Effect on Symmetry Breaking by Wilson Loops
}

\author{
Kiyoshi Shiraishi \\ Department of Physics, Tokyo Metropolitan University, \\ Setagaya, Tokyo, 158 Japan
}

Zeitschrift für Physik C35 (1987) pp. 37-42

\begin{abstract}
A finite temperature and density effect of Wilson loop elements on non-simply connected space is investigated in the model suggested by Hosotani. Using one-loop calculations it is shown that the value of an "order parameter" does not shift as the temperature grows. We find that finite density effect is of much importance for restoration of symmetry.
\end{abstract}

\section{Introduction}

For the unification of interactions between elementary particles there has been a renewed interest in higher-dimensional theories. In particular recent development of superstring theories [1] attracts much attention of many particle physicists. The development has been prompted by the discovery of anomaly-free nature of the Green-Schwartz string theory [2]. Soon heterotic string was constructed by Gross et al. [3]; that is the string theory with gauge symmetry group $E_{8} \times E_{8}$ or $O(32) / Z_{2}$. The gauge group $E_{8} \times E_{8}$ is sufficient for incorporating the observed fundamental gauge interactions. Thus many string phenomenologists investigate the so-called "superstring-inspired models" [4] and there has been detailed discussion on them.

Contrary to the "pure" Kaluza-Klein concept [5], the gauge symmetry must be broken, rather than be generated, to the one favored phenomenologically when extra dimensions are compactified. Symmetry breaking is expected to be caused by Wilson loop elements on non-simply connected internal space in consistent with supersymmetry $[6,7]$; incidentally non-simply connected space is required to reduce the number of fermion families [6].

Somewhat similar mechanisms have been used previously by Hosotani [8] and Toms [9]. Recently Midorikawa and Tomiya [10] have shown the detailed implication of the symmetry breaking mechanism at "classical" level. In the models considered by the above authors, a constant vacuum gauge field is allowed on the multiply-connected space; the vacuum expectation value of the gauge field 
configuration plays the role of an "order parameter". Moreover Hosotani has considered a simple model to show that one-loop vacuum effect determines the order parameter [8]. Evans and Ovrut have calculated the vacuum energy splitting of gauge field vacua on various multiply connected manifolds [11].

Apart from string theories, an interesting unified model with $S U(5)$ symmetry using Hosotani's mechanism was suggested by Svetovoi and Khariton [12], which naturally explained the existence of light Higgs doublet. Although there has been no realistic model, Hosotani's mechanism may give a new aspect of symmetry breaking in the unified model with and without supersymmetry.

On the other hand, for modern cosmologists, the phase transition in the history of our universe is a very important issue [13]. In ordinary field theories including spontaneous symmetry breaking, the symmetry is expected to be restored at high temperature [14] or high density [15]. The finite temperature effect is also important for an investigation of stable compactification in Kaluza-Klein theories [16].

In the present paper, we consider the finite temperature and density effect on symmetry breaking mechanism proposed by Hosotani. In Sect. II, we discuss Hosotani's model and the mass levels of the particles obtained by compactification. In Sect. III, we consider the effective potential at finite temperature, namely, the free energy. Finite density effect of fermions is considered in Sect. IV. Finally, Sect. V is devoted to discussion.

\section{The Wilson Loop on $S^{1}$ and the Mass Spec- trum}

In this paper, we concentrate our attention to Hosotani's model in four dimensions [8]. Further, for simplicity, we treat the $S U(2)$ gauge symmetry[10].

First suppose that the spacetime topology is $M_{3} \times S^{1}$ where $M_{3}$ is a three dimensional Minkowski space-time and $S^{1}$ is a circle whose circumference is $L$. The four dimensional coordinates are represented by $(x, y)$, where $0 \leq y<L$.

Let us consider $S U(2)$ gauge theory in this space-time; for the present, we take $S U(2)$ gauge fields $A_{\mu}$ and fundamental massless fermions $\psi \cdot S^{1}$ is a compact and non-simply connected space, i.e., $\Pi_{1}\left(S^{1}\right)=Z$. Boundary conditions on $A_{\mu}$ and $\psi$ are chosen definitely as follows:

$$
\left\{\begin{array}{l}
A_{\mu}(x, y+L)=A_{\mu}(x, y) \\
\psi(x, y+L)=e^{i \delta} \psi(x, y)
\end{array} .\right.
$$

At this level, symmetry breaking is not observed at all.

On the multiply-connected manifold, non zero vacuum gauge configuration is permitted. In our case, we set

$$
\left\langle g L A_{y}\right\rangle=\frac{1}{2}\left[\begin{array}{cc}
\phi & 0 \\
0 & -\phi
\end{array}\right]=\frac{\phi}{2} \tau_{3},
$$

where the vacuum gauge field has been diagonalized by using the freedom of gauge transformations and $g$ is the gauge coupling constant. If we just consider 
non-singular gauge transformation, $\left\langle g L A_{y}\right\rangle$ is inequivalent to the trivial vacuum gauge field $\left\langle g L A_{y}\right\rangle=0$ unless $\left\langle g L A_{y}\right\rangle=2 \pi n \tau_{3}$ (n: integer). We can only restrict $0 \leq \phi<4 \pi$ by gauge transformations. The value of a Wilson loop element is:

$$
U=P \exp \left(-i g \int_{\gamma} A_{y} d y\right)=\left[\begin{array}{cc}
e^{-i \frac{\phi}{2} n} & 0 \\
0 & e^{i \frac{\phi}{2} n}
\end{array}\right],
$$

where the closed path $\gamma$ winds around $S^{1} n$ times. Note that $U$ is regarded as an element of the additive group $Z\left(=\Pi_{1}\left(S^{1}\right)\right)$.

On a general multiply-connected manifold $U \neq 1$ necessarily; moreover, on certain manifolds, $U$ is taken to be an element of a finite group, then we can tell the symmetry breaking pattern by classification of $U$.

Now in our model, in view of the three-dimensional flat space, one finds ladders of the discrete mass levels of infinite number of fields by Fourier expansions in terms of $y$; those masses are:

$$
\frac{1}{L}(2 \pi n+\phi) \quad(n: \text { integer })
$$

which come from the expansion of gauge fields $A_{\mu}^{1}$ and $A_{\mu}^{2}$

$$
\frac{1}{L}(2 \pi n) \quad(n: \text { integer })
$$

which come from the expansion of $A_{\mu}^{3}$ and

$$
\frac{1}{L}\left(2 \pi n \pm \frac{\phi}{2}-\delta\right) \quad(n: \text { integer })
$$

which come from the expansion of fermions.

In general, $\phi$ lifts the mass level; this effect reduces the number of the massless fields in three dimension within the Kaluza-Klein point of view. In the next section, we will see how the value of $\phi$ is determined by one-loop quantum effect.

\section{One-Loop Quantum Effect and Finite Tem- perature}

In order to calculate the one-loop effective potential for the model, we use a covariant derivative with constant gauge fields. For instance, one finds the expression of the effective potential for fundamental fermion fields:

$$
V_{\text {eff }}=-\frac{N_{F}}{(\text { Volume })} \ln \operatorname{det}(\not D),
$$

where

$$
D_{\mu}=\partial_{\mu}-i g A_{y} \delta_{y \mu} .
$$


$N_{F}$ is the number of the fermion fields which belong to a fundamental representation of $S U(2)$.

Of course, we also take care of the compactness of $S^{1}$ in the calculation. Thus, the effective potential for the system under consideration is given by [8] :

$$
V_{e f f}=-\frac{2}{L^{4} \pi^{2}} \sum_{\ell=1}^{\infty} \frac{1}{\ell^{4}}[2 \cos (\ell \phi)+1]+N_{F} \frac{4}{L^{4} \pi^{2}} \sum_{\ell=1}^{\infty} \frac{1}{\ell^{4}} 2 \cos (\delta \ell) \cos \left(\ell \frac{\phi}{2}\right),
$$

where $0 \leq \phi<4 \pi$. The first term comes from the contribution of $S U(2)$ gauge fields and the other from fermions.

As Hosotani [8] has pointed out, an absolute minimum of $V_{\text {eff }}$ depends on $\delta$; that is

$$
\begin{aligned}
\langle\phi\rangle & =2 \pi \text { for }|\delta| \leq \pi / 2 \\
\text { and } & \\
\langle\phi\rangle & =0 \text { for } \pi / 2 \leq|\delta| \leq \pi .
\end{aligned}
$$

When $|\delta|<\pi / 2$, though the gauge symmetry is not broken, the fermion masses in three dimensions become

$$
\frac{1}{L}(2 \pi n+\pi-\delta) \quad(n \text { : integer }) .
$$

Even if one imposes $\delta=0$, the result yields the antiperiodic boundary condition i.e. there is no massless fermion in the three dimensions.

We can interpret this result as the breakdown of a $Z_{2}$ symmetry [17]. In the model of this type for $S U(N)$, the breakdown of a $Z_{N}$ symmetry may occur by introducing fermions.

Hosotani [8] also pointed out that symmetry breakdown from $S U(2)$ to $U(1)$ may take a place with putting scalar bosons of fundamental representation into the system. From now on, when we say "symmetry" it means those of $Z_{N}$ as well as $S U(N)$. Detailed issues, such as the connection between gauge transformations and the boundary condition on gauge fields, cannot be treated here. One finds an extensive discussions in $[8,9,10]$.

Now, we shall consider the finite temperature effect. In the one-loop approximation, we perform a calculation of "free energy" at temperature $T=\beta^{-1}$ similarly to the derivation of the zero-temperature effective potential; only one difference is that we must impose boundary conditions on fields with respect to the Euclidean time coordinate $\tau(0 \leq \tau<\beta)$, i.e., periodic for bosons and antiperiodic for fermions [14]. It is well known that these conditions lead naive particle statistics.

At carrying out the calculation, the regularization is needed as we used in the zero-temperature case; here, we simply drop the divergence independent of $\phi$. Consequently, the free energy $\Omega$ is obtained as follows:

$$
\Omega=-\frac{2 V L}{L^{4} \pi^{2}}\left[\frac{\pi^{4}}{30}\left(\frac{L}{\beta}\right)^{4}+\frac{\pi^{4}}{90}+2 \sum_{\ell=1}^{\infty} \frac{\cos \ell \phi}{\ell^{4}}\right.
$$




$$
\begin{aligned}
& \left.+4 \sum_{n=1}^{\infty} \sum_{\ell=1}^{\infty} \frac{\cos \ell \phi}{\left(\frac{\beta^{2}}{L^{2}} n^{2}+\ell^{2}\right)^{2}}+2 \sum_{n=1}^{\infty} \sum_{\ell=1}^{\infty} \frac{1}{\left(\frac{\beta^{2}}{L^{2}} n^{2}+\ell^{2}\right)^{2}}\right] \\
& +N_{F} \frac{4 V L}{L^{4} \pi^{2}}\left[-\frac{7}{8} \frac{\pi^{4}}{90}\left(\frac{L}{\beta}\right)^{4}+\sum_{\ell=1}^{\infty} \frac{1}{\ell^{4}} \cos (\delta \ell) \cos \left(\ell \frac{\phi}{2}\right)\right. \\
& \left.+2 \sum_{n=1}^{\infty} \sum_{\ell=1}^{\infty} \frac{(-1)^{n}}{\left(\frac{\beta^{2}}{L^{2}} n^{2}+\ell^{2}\right)^{2}} \cos (\delta \ell) \cos \left(\ell \frac{\phi}{2}\right)\right]
\end{aligned}
$$

where $V$ is the two dimensional volume of the system.

At a glance, one finds a peculiarity of the symmetry breaking mechanism; the expression for the free energy of the system consists of the trigonometrical functions. It is expected that possible extrema of the free energy locate at the peculiar values of $\phi$ (i.e., $0, \pi, 2 \pi$ etc.) since the coefficient of the "highfrequency-wave" functions are well suppressed by a factor $\sim \ell^{-D}$ or $n^{-D}$, where $D$ is the total dimensions of the space-time.

Now we consider the high temperature limit $(\beta / L \rightarrow 0)$. Using asymptotic forms of the following numerical series,

$$
\begin{aligned}
& \sum_{n=-\infty}^{\infty} \frac{1}{\left(n^{2}+a^{2}\right)^{2}}=\frac{\pi}{2} \frac{1}{a^{3}}+O\left(e^{-2 \pi a} / a^{3}\right) \\
& \sum_{n=-\infty}^{\infty} \frac{(-1)^{n}}{\left(n^{2}+a^{2}\right)^{2}}=O\left(e^{-\pi a} / a^{3}\right) \quad(a \gg 1),
\end{aligned}
$$

we can approximate the free energy (13) at $\beta / L \ll 1$ :

$$
\Omega \sim-V L \frac{\pi^{2}}{90}\left(2 \times 3+\frac{7}{8} \times 4 \times N_{F}\right) \frac{1}{\beta^{4}}-\frac{2 V L}{L^{3} \beta}\left[2 \sum_{\ell=1}^{\infty} \frac{\cos (\ell \phi)}{\ell^{3}}+\zeta(3)\right] .
$$

Accordingly, we observe that the $\phi$-dependent part of the free energy (while the rest is pure radiation) which comes from gauge fields becomes dominant in the high temperature limit; it has degenerate minima at $\phi=0$ and $2 \pi$.

Though the fermionic contribution becomes much suppressed at high temperature, a careful evaluation reveals that the location of the minima is not changed from the values at zero temperature; at high temperature the contribution from "high frequency" trigonometrical functions are exponentially suppressed.

After all, it is found that there is no change of symmetry pattern, i.e., symmetry restoration or breakdown (of $Z_{2}$ ) in this system does not occur by raising temperature.

If one wants to make a system whose symmetry depends on temperature, we must manage the combination of bosons and fermions with various representations in general gauge theories. For example, we suppose an $S U(2)$ gauge 
theory with $N_{F}$ fermions and $N_{S}$ scalar bosons which are both in fundamental representation. Further, if we impose $\delta \sim \pi / 2$ as boundary conditions on both fields and set $N_{S}=2 N_{F} \gg 1$, one finds the gauge symmetry breaking by raising temperature of the system.

To summarize, the extrema of the free energy, or effective potential in terms of $\phi$ is almost insensitive to finite temperature effect. To make the model whose symmetry pattern depends on temperature, we must make use of the difference of statistics between bosons and fermions. These properties are held in models with Hosotani's mechanism.

\section{Effect of Finite Density and the Degenerate Fermions}

In this section, we investigate a finite density effect on the Hosotani's model which has been treated previously. For this purpose, we employ the chemical potential for fermions; at the same time, the free energy is extended to the "thermodynamic potential". A prescription of deriving the expression for the thermodynamic potential at one-loop level is already written down [18]; in a course of the calculation the covariant derivative need to be modified as

$$
D_{0}=\partial_{0} \rightarrow \partial_{0}-i A_{0}, \quad \text { where } A_{0}=-i \mu .
$$

Thus we only carry out the usual one-loop calculation as there is a constant but imaginary $U(1)$ gauge potential in zeroth component. It is interesting to find a similarity in the one-loop calculation for compact space and the periodic time direction.

Finite density effect on various symmetry breakdown mechanisms have been studied extensively [15]. In the Kaluza-Klein context, the thermodynamic potential with $\mu \neq 0$ was discussed by the present author [19].

The calculation technique is parallel to the one used in [19]. The expression is given in Appendix. Here, we consider the strongly degenerate fermi gas, i.e., in the situation of $\mu \neq 0$ and $T \rightarrow 0$. For simplicity, we shall give the expression for the thermodynamic potential of fermions in fundamental representation of $S U(2)$ with periodic boundary condition $(\delta=0)$ on $S^{1}$.

Within the Kaluza-Klein point of view, it is interesting to study the case that $\mu$ is less than, or at most nearly equals to $2 \pi / L$. Accordingly, we only consider the case that $0<\mu L<\pi$ here.

The thermodynamic potential, or the effective potential of $\phi$ with $\mu \neq 0$, is given by:

$$
\begin{aligned}
\Omega= & N_{F} V L \frac{1}{L^{4} \pi^{2}}\left[\frac{1}{6}\left\{2 \pi^{2}\left(\frac{\phi}{2}-\pi\right)^{2}-\left(\frac{\phi}{2}-\pi\right)^{4}-\frac{7}{15} \pi^{4}\right\}\right. \\
& \left.-\frac{\pi}{3}\left(\frac{\phi}{2}-\mu L\right)^{2}(\phi+\mu L)\right] \\
& \text { for } 0<\phi / 2 \leq \mu L,
\end{aligned}
$$




$$
\begin{aligned}
\Omega= & N_{F} V L \frac{1}{L^{4} \pi^{2}}\left[\frac{1}{6}\left\{2 \pi^{2}\left(\frac{\phi}{2}-\pi\right)^{2}-\left(\frac{\phi}{2}-\pi\right)^{4}-\frac{7}{15} \pi^{4}\right\}\right. \\
& \text { for } \mu L<\phi / 2 \leq 2 \pi-\mu L, \\
\Omega= & N_{F} V L \frac{1}{L^{4} \pi^{2}}\left[\frac{1}{6}\left\{2 \pi^{2}\left(\frac{\phi}{2}-\pi\right)^{2}-\left(\frac{\phi}{2}-\pi\right)^{4}-\frac{7}{15} \pi^{4}\right\}\right. \\
& \left.-\frac{\pi}{3}\left(2 \pi-\frac{\phi}{2}-\mu L\right)^{2}(4 \pi-\phi+\mu L)\right] \\
& \text { for } 2 \pi-\mu L<\phi / 2 \leq 2 \pi,
\end{aligned}
$$

An absolute minimum of $\Omega$ is given by $\phi / 2=\pi$ for $\mu L<2^{-1 / 3} \pi$ and $\phi / 2=0$ for $2^{-1 / 3} \pi<L<\pi$. For $\mu L=2^{-1 / 3} \pi$ these two minima are degenerate.

One can find that the non-zero density of fermion may restore the symmetry. It is remarkable that finite density effect is much important for symmetry breaking while finite temperature has almost no effect on it.

In the region $\mu L>\pi$, for sufficient large $\mu L(>1.9 \pi)$ the point $\phi / 2=\pi$ becomes an absolute minimum again; moreover, for $\mu L>2 \pi$, new minima whose locations depend on $(\mu L)$ even appear. Furthermore, in the region $\mu L \gg 2 \pi$, finding of the vacuum requires rather complicated investigations.

The analysis of symmetry pattern will be more complicate if we consider the model with realistic larger gauge group.

\section{Discussion}

In this paper, we calculate the one-loop effective potential at finite temperature and density, or the thermodynamic potential, for the simple model suggested by Hosotani [8] and investigate its symmetry breaking pattern. It turns out that the degenerate fermions have much influence on the symmetry breaking through the thermodynamic potential, while the finite temperature only gives the correction which has almost the same shape as the effective potential at $T=0$.

Therefore, we should look for the evidence of the compact dimensions inside the cold fermion star as well as near the black holes [20]! Actually, however, only "theoretical" searches can be done for simple models, one of which is examined in this paper. In order to use the Hosotani's mechanism in unified theories, we must correctly of course take into account the gravitational effects and, realistic gauge group and compact manifold.

$S^{1}$, the multiply-connected space, is a rather exceptional one. In the context of compactified string models, the manifold $M$ written as $M=K / H$ where $K$ is a simply-connected manifold and $\mathrm{H}$ is a finite group is often considered; then $\Pi_{1}(K / H)=H$. In this case, a Wilson loop can be regarded as an element of a finite group $H$, while $\Pi_{1}\left(S^{1}\right)=Z$ is an infinite group. One-loop vacuum energy has been calculated by Evans and Ovrut [11] for some simple cases such as 
$M=S^{3} / Z_{2}$. The method of the calculation is fairly different from the one for the model in the present paper, because symmetry of the finite group restricts the eigenmode on $M$. It is also important to consider the finite temperature and density effect on such models. Because the temperature and/or density may break the supersymmetry, it is of interest to study a model with various particle content.

On the other hand, the presence of gauge fields implies the existence of conserved quantities in the system. Moreover, the asymptotic-free nature of non-Abelian gauge theory imposes the "colorlessness" of the system. The effect have been considered in the investigation of the "quark-gluon plasma" [21]. The colorless partition function can be obtained by averaging the naive partition function in terms of the zeroth component of the vacuum gauge fields; i.e., the covariant derivative $D_{0}=\partial_{0}-i \mathbf{A}_{0}$ is employed to derive the one-loop result. It is naturally expected that the global color confinement and the non-trivial Wilson loop element on multiply-conected space are influenced by each other as is in the case of $\mu \neq 0$.

Imposing the color singlet condition may be of great relevance not only to the dynamics in the very early universe as is pointed out in [22] but also the Kaluza-Klein cosmology, on account of shrinking extra dimensions.

However, since matter fields play both roles to break the symmetry and to spoil the confinement, the matter content of the theory must be specified within certain extent for a detailed analysis. Therefore we have left the discussion on the effect of the "colorlessness" condition with the symmetry breaking by Wilson loops until more realistic models appear.

\section{Acknowledgement}

The author would like to thank M. Hosoda for reading this manuscript and useful comments.

\section{Appendix}

Here, we shall briefly show the calculation of the thermodynamic potential treated in the text. In the space $R^{d} \times S^{1}$, the thermodynamic potential for $S U(2)$ doublet fermions with $\mu \neq 0$ and $\delta=0$ is formally expressed as follows:

$$
\begin{aligned}
& \frac{4 N_{F}}{\beta} \frac{V}{(2 \pi)^{d}} \int_{0}^{\infty} d t t^{-1} \int d^{d} k \sum_{n=-\infty}^{\infty} \sum_{\ell=-\infty}^{\infty} \\
& \cdot \operatorname{Re}\left[\exp \left\{-t\left(k^{2}+\left(\frac{(2 n+1) \pi}{\beta}+i \mu\right)^{2}+\left(\frac{2 \pi \ell+\phi / 2}{L}\right)^{2}\right)\right\}\right] \\
& (0 \leq \phi / 2<2 \pi) .
\end{aligned}
$$


After dropping the divergence which is independent of $\phi$, it becomes:

$$
\begin{gathered}
\Omega=4 N_{F} V L\left[\frac{1}{\pi^{\frac{d+2}{2}}} \Gamma\left(\frac{d+2}{2}\right) \cdot 2 \sum_{\ell=1}^{\infty} \frac{1}{(L \ell)^{d+2}} \cos \left(\ell \frac{\phi}{2}\right)\right. \\
+\frac{1}{L(4 \pi)^{d / 2}} 4 \sum_{n=1}^{\infty}(-1)^{n} \cosh (\mu \beta n)\left\{\left(\frac{\phi}{L \beta n}\right)^{\frac{d+1}{2}} K_{\frac{d+1}{2}}\left(\frac{\beta \phi}{2 L} n\right)\right. \\
+\sum_{\ell=1}^{\infty}\left[\left(\frac{2(2 \pi \ell+\phi / 2)}{L \beta n}\right)^{\frac{d+1}{2}} K_{\frac{d+1}{2}}\left(\beta n \frac{2 \pi \ell+\phi / 2}{L}\right)\right. \\
\left.\left.\left.+\left(\frac{2(2 \pi \ell-\phi / 2)}{L \beta n}\right)^{\frac{d+1}{2}} K_{\frac{d+1}{2}}\left(\beta n \frac{2 \pi \ell-\phi / 2}{L}\right)\right]\right\}\right] .
\end{gathered}
$$

(Here, we used some identities which can be found in [19].)

In order to find the expression in the case of $T \rightarrow 0(\beta \rightarrow \infty)$, we use an integral representation of the modified Bessel function:

$$
K_{\nu}(z)=\frac{\sqrt{\pi}(z / 2)^{\nu}}{\Gamma(\nu+1 / 2)} \int_{1}^{\infty} e^{-z x}\left(x^{2}-1\right)^{\nu-1 / 2} d x .
$$

Using this, we can perform the summation over $n$ to obtain:

$$
\begin{aligned}
\Omega & =4 N_{F} V L\left[\frac{1}{\pi^{\frac{d+2}{2}}} \Gamma\left(\frac{d+2}{2}\right) \cdot 2 \sum_{\ell=1}^{\infty} \frac{1}{(L \ell)^{d+2}} \cos \left(\ell \frac{\phi}{2}\right)\right. \\
& -\frac{1}{L} \frac{1}{(4 \pi)^{d / 2}} \frac{1}{\Gamma\left(\frac{d+2}{2}\right)}\left\{\left(\frac{\phi}{2 L}\right)^{d+1} \int_{1}^{\infty} d x\left(x^{2}-1\right)^{d / 2}\right. \\
& \cdot\left(\frac{1}{\exp \left[\beta\left(\frac{\phi}{2 L} x-\mu\right)+1\right]}+(\mu \rightarrow-\mu)\right) \\
& +\sum_{\ell=0}^{\infty}\left\{\left[\left(\frac{2 \pi \ell+\phi / 2}{L}\right)^{d+1} \int_{1}^{\infty} d x\left(x^{2}-1\right)^{d / 2}\right.\right. \\
& \left.\left.\left.\cdot\left\{\frac{1}{\exp \left[\beta\left(\frac{2 \pi \ell+\phi / 2}{L} x-\mu\right)+1\right]}+(\mu \rightarrow-\mu)\right\}+[\phi \rightarrow-\phi]\right\}\right\}\right]
\end{aligned}
$$

In the limit of $\beta \rightarrow \infty$,

$$
\frac{1}{e^{\beta x}+1} \stackrel{\beta \rightarrow \infty}{\longrightarrow} \theta(-x)
$$

is recognized, and at the same time an infinite series becomes a finite sum over $\ell$.

Soon we can find the expressions $(18,19,20)$ in the text when $d=2$, $0<\mu L<\pi$. 


\section{References}

[1] Selected papers can be found in: J. Schwarz (ed.), Superstrings (Singapore: World Scientific 1985).

[2] M. Green and J. Schwarz, Phys. Lett. B149 (1984) 119.

[3] D. Gross, J. Harvey, E. Martinec and R. Rohm, Phys. Rev. Lett. 54 (1985) 502; Nucl. Phys. B256 (1985) 253; Nucl. Phys. B267 (1986) 75.

[4] J. Ellis, K. Enqvist, D. V. Nanopoulos and F. Zwirner, Nucl. Phys. B276 (1986) 14.

[5] Th. Kaluza, Sitzungsber. Preuss. Akad. Wiss., Berlin, Math. Phys. K1 (1921) 966; O. Klein: Z. Phys. 37 (1926) 895; E. Witten, Nucl. Phys. B186 (1981) 412, and references therein.

[6] P. Candelas, G. Horowitz, A. Strominger and E. Witten, Nucl. Phys. B258 (1985) 46.

[7] E. Witten, Nucl. Phys. B258 (1985) 75; J. Breit, B. Ovrut and G. Segrè, Phys. Lett. B158 (1985) 33; A. Sen, Phys. Rev. Lett. 55 (1985) 33.

[8] Y. Hosotani, Phys. Lett. B126 (1983) 309.

[9] D. Toms, Phys. Lett. B126 (1983) 445.

[10] S. Midorikawa and M. Tomiya, Preprint INS-Rep.-597 (July 1986).

[11] M. Evans and B. A. Ovrut, Phys. Lett. B174 (1986) 63; B.A. Ovrut, Prog. Theor. Phys. Suppl. 86 (1986) 185.

[12] V.B. Svetovoi and N.G. Khariton, Sov. J. Nucl. Phys. 43 (2) (1986) 280.

[13] For example, see Nucl. Phys. B252, Nos. 1, 2 (1985).

[14] D. A. Kirzhnits and A. D. Linde, Phys. Lett. B42 (1972) 471; S. Weinberg, Phys. Rev. D9 (1974) 3357; L. Dolan and R. Jackiw, Phys. Rev. D9 (1974) 3320; A. D. Linde, Rep. Prog. Phys. 42 (1979) 389.

[15] D. Bailin and A. Love, Nucl. Phys. B226 (1983) 493; B233 (1984) 204; A. Love and S. J. Stow, Nucl. Phys. B243 (1984) 537; B257 [FS14] (1985) 271.

[16] F. S. Accetta and E.W. Kolb, Phys. Rev. D34 (1986) 1798, and references therein.

[17] N. Weiss, Phys. Rev. D25 (1982) 2667.

[18] A. Actor, Nucl. Phys. B265 [FS15] (1986) 689, and references therein.

[19] K. Shiraishi, Prog. Theor. Phys. 77 (1987) 1253. 
[20] A. Davidson and D.A. Owen, Phys. Lett. B155 (1985) 247; M. Yoshimura, Preprint KEK-TH 116 (1985); Phys. Rev. D34 (1986) 1021; S. Midorikawa and M. Tomiya, Preprint INS-Rep. 603 (1986).

[21] B. Müller, The physics of the quark-gluon plasma (Berlin, Heidelberg, New York, Springer 1985); H. T. Elze and W. Gleiner, Phys. Rev. A33 (1986) 1879 , and references therein.

[22] B. S. K. Skagerstam, Phys. Lett. B133 (1983) 419. 